\title{
Explicit-Ready Nonlinear Model Predictive Control for Turbocharged Spark- Ignited Engines
}

\author{
J. El Hadef ***, G. Colin*, Y. Chamaillard*, S. Olaru***, P. Rodriguez-Ayerbe***, V. Talon** \\ *Laboratoire PRISME, 8 rue Léonard de Vinci, 45072 Orléans cedex 2, France \\ (Tel: +33238494383; e-mail: jamil.el-hadef@etu.univ-orleans.fr). \\ **Renault SA - CTL, 1 Allée de Cornuel, 91510 Lardy, France (e-mail: vincent.talon@renault.com) \\ ***E3S (Supelec System Science), Automatic Control Department, France (email : sorin.olaru@supelec.fr)
}

\begin{abstract}
The trend towards reducing engine size in the automotive industry, motivated by more restrictive pollutant emission standards, has led to increasingly complicated engine technical definitions. The control challenge has also grown since engines are now considered as highly nonlinear multi-input multi-output systems with saturated actuators. In this context, the need for model-based control laws is greater than ever. In this study we propose a nonlinear model predictive control strategy based on a physical engine model. Moreover, we also underline the benefit of using a thermodynamic engine term in the objective function. Finally, the design and calibration choices consciously fulfill the criteria of the use of an explicit approach for real time implementation.
\end{abstract}

\section{INTRODUCTION}

Objectives in terms of pollutant emissions and fuel economy have led car manufacturers to complicate the technical definitions of both gasoline and diesel engines. Modern combustion engines can now be defined as multi-input multioutput nonlinear systems with saturated actuators. In this paper, we evaluate the benefits of a nonlinear model predictive control (NMPC) law in terms of pressure set point tracking performances, calibration efforts and engine efficiency optimization. The study is motivated by the fact that model predictive control (MPC) is already well established in various industries for controlling multivariable processes (Camacho et al., 2004). Until now, calculation time considerations have prevented it from penetrating the automotive industry. Recently, however, so-called explicit model predictive control has broken this limit and enlarged the range of possible applications (Bemporad et al., 2000, Del Re et al., 2010, Di Cairano et al., 2007, Grancharova et al., 2012).

In this study, a nonlinear predictive controller, using a physics-based engine model, has been designed and calibrated. The model relies on the so-called zerodimensional (0D) modeling approach (Eriksson, 2007, Eriksson et al., 2002) in order to maintain a low calculation time while benefiting from the accuracy of a simulator-like engine model. We also propose a new objective function which optimizes the engine efficiency by explicitly reducing the pumping losses. The comparison with a more classical method which consists in optimizing the throttle opening is presented. Conclusions stress the advantages of the new cost function, in particular for more advanced engine technical definitions. It should also be noted that the NMPC law that is presented in this paper has been designed to be implemented online using the explicit approach. As such, it fulfills all the criteria that are required to do so.

The paper is organized as follows. Section 2 presents the system and the control objectives. Section 3 presents insights into the physics-based model of a turbocharged spark-ignited (SI) engine. The NMPC law and two objective functions are detailed in section 4. Simulation results are presented in section 5. The conclusion summarizes the main outcome of the study and the next steps to be achieved.

\section{SYSTEM DESCRIPTION}

\subsection{Turbocharged SI engine}

The purpose of this study is to control the air path of a $1.2 \mathrm{~L}$ turbocharged SI engine (Fig. 1).

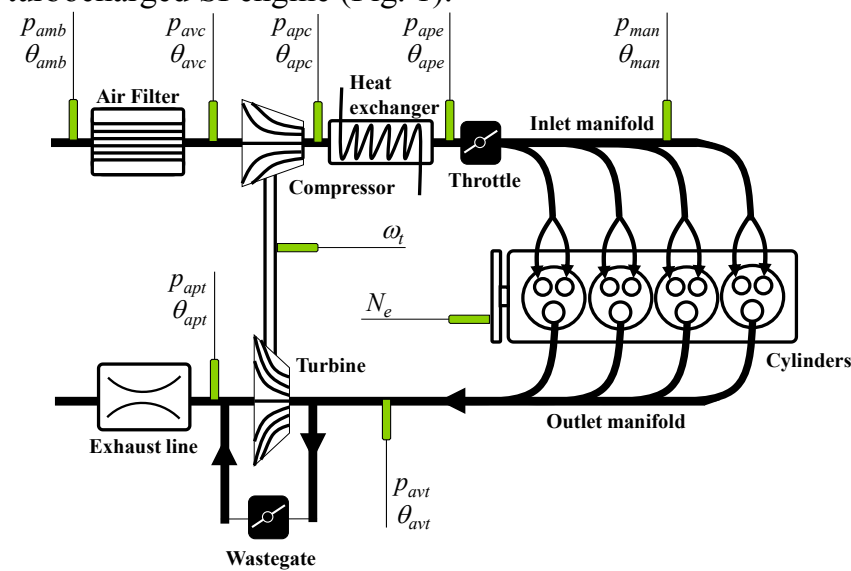

Fig. 1. Air path sketch of a turbocharged SI engine ( $p$ stands for pressure, $\theta$ for temperature, $N_{e}$ and $\omega_{t}$ are respectively the engine and turbocharger rotational speed).

At the intake, a compressor and a heat exchanger successively increase the pressure and cool down the fresh air flow. Then, a variable flow restriction, called throttle, controls the inlet manifold pressure $p_{\text {man }}$. At the exhaust, the 
amount of gas which passes through a turbine is controlled by a by-pass, known as a wastegate (Fig. 1). This energy, recovered at the exhaust, drives the intake compressor and its outlet boost pressure $p_{a p c}$ (Heywood, 1988).

\subsection{Control objectives}

In order to maximize the efficiency of the three-way catalytic converter, SI engines operate at stoichiometric conditions, i.e. the air/fuel equivalent ratio is always equal to one. Consequently, from the control point of view, the engine torque is directly controlled by the air mass entering the cylinders. For a given engine speed, this mass directly depends on the inlet manifold pressure ratio. A static map, calibrated on test bench measurements, is usually used to build the inlet manifold pressure reference trajectories from the engine torque set point.

The objective of the air path controller is to determine which throttle and wastegate positions will achieve this pressure in the inlet manifold. Since there is an infinite number of actuator positions to achieve a given inlet manifold pressure, one would like to select the most efficient one. Usually, this multi-input and multi-objective control problem is divided into two single-input single-objective problems (Moulin et al., 2008). The throttle is used to achieve an inlet manifold pressure reference trajectory and the wastegate is used to control the compressor outlet pressure. The rule of thumb to maximize the efficiency is to use the same set point (Colin, 2006), i.e.:

$$
p_{\text {ape }}^{S P}=p_{\text {man }}^{S P}
$$

In these conditions, the throttle opening is maximized and intuitively the use of the turbocharger is minimized. Since the use of the turbine creates a flow restriction at the exhaust, the engine efficiency is expected to increase. In fact, the link between the exhaust pressure and the engine efficiency can be explained on the cylinder pressure-volume $(\mathrm{P}-\mathrm{V})$ diagram (Colin, 2006) of four-stroke engines (Fig. 2).

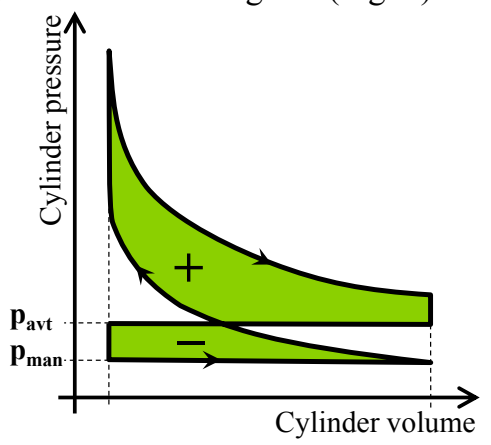

Fig. 2. Theoretical P-V diagram in the cylinder of a four stroke internal combustion engine. The upper loop represents the work produced by the engine. The bottom loop represents the work consumed by the engine to suck up the air from the inlet manifold, i.e. the pumping losses.

The area of the second loop, which represents losses, is directly linked to the difference between the exhaust manifold pressure $p_{a v t}$ and the inlet manifold pressure $p_{\text {man }}$. Since the inlet manifold pressure is given by the torque set point, reducing the exhaust pressure is the only way to reduce the pumping losses and increase the engine efficiency.

However, because of the turbocharger thermodynamic properties, maximizing the throttle opening does not always lead to minimizing the exhaust pressure. In particular, a better turbocharger operating point, in terms of exhaust pressure, could exist with a smaller throttle opening. In this paper, we propose to overcome this problem by directly using the thermodynamic criterion deduced from the $\mathrm{p}-\mathrm{V}$ diagram. It consists in minimizing the ratio between the exhaust pressure and the inlet manifold pressure in order to reduce the area of the bottom loop. The advantage of such a method is that it guarantees that the engine will operate at its best efficiency point.

\section{PHYSICS-BASED 0D ENGINE MODEL}

Models that are used in MPC must capture both static and dynamic behaviors of the system (Camacho et al., 2004). For real-time automotive control, authors usually select mathematical models such as state-space representations (Del Re et al., 2010). They allow fast output prediction but usually require numerous test bench measurements to be calibrated. Moreover, since combustion engines are in fact highly nonlinear, multiple local linear models are usually required to cover the entire operating range (Colin, 2006, Pekar et al., 2012).

In an explicit approach, the online computational time is not linked to the model complexity since it is only used offline (Bemporad et al., 2000, Grancharova et al., 2012). For this reason, we propose to use a single nonlinear model. Among all the nonlinear approaches, physics-based models lead to improved prediction results. Additionally, they allow extrapolation to new operating points while maintaining a low calibration effort. In order to bring the offline computation effort under control, a 0D modeling approach combined with a mean value cylinder model was chosen (Moulin et al., 2008). The model is succinctly described below but more details can be found in El Hadef et al., 2012a, Eriksson, 2007, Eriksson et al., 2002, Heywood, 1988.

\subsection{Hypothesis and modelling philosophy}

The air path is discretized: a control volume of the air path is followed by a flow restriction, itself followed by another control volume and so forth (Fig. 3).

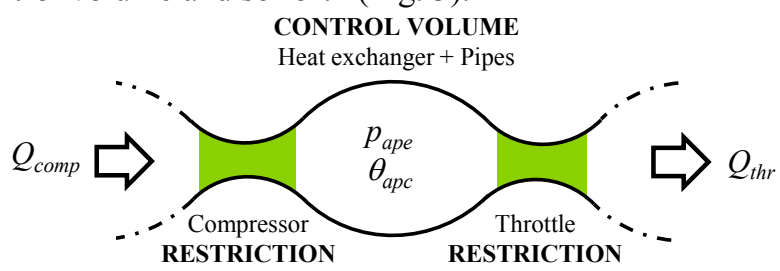

Fig. 3. Example of a succession of control volumes and restrictions: the heat exchanger and its pipes are surrounded by two flow restrictions: the compressor and the throttle.

In each control volume, the pressure and the temperature describe the complete thermodynamic state of the volume. In order to reduce the number of states of the model, the 
temperature dynamics can be neglected (Hendricks, 2001). In that case, the temperatures are computed through simple algebraic relations. Pressure behavior is governed by a differential equation deduced from Euler's mass, energy and momentum equations (Eriksson, 2007, Eriksson et al., 2002). Altogether, the model contains three control volumes: the inlet and outlet manifolds and the heat exchanger (Fig. 1). It respectively corresponds to three states: $p_{\text {man }}, p_{\text {avt }}$ and $p_{\text {ape }}$. In order to take into account the dynamics of the turbocharger, a fourth state is required: the turbocharger rotational speed $\omega_{t}$.

\subsection{Pressures in the three control volumes}

Under the assumption of constant temperature $\theta$ in the volume $\mathrm{V}$, the pressure time derivative $\frac{\partial p}{\partial t}$ is given by:

$$
\frac{\partial p}{\partial t}=\frac{\gamma r}{V}\left(Q_{m_{\text {in }}} \theta_{\text {in }}-Q_{m_{\text {out }}} \theta_{\text {out }}\right)
$$

where $\gamma$ is the ratio of specific heat, $r$ is the fluid gas constant and $Q_{m}$ the mass flow rate. Indices "in" and "out" respectively stand for inlet and outlet of the considered control volume.

\subsection{Engine mean value model}

Because of acoustic phenomena and valve phasing, the theoretical flow entering the cylinders at manifold conditions is corrected by a volumetric efficiency coefficient $\eta_{v o l}$ :

$$
Q_{\text {eng }}=\frac{p_{\text {man }} V_{c y l}}{r \theta_{\text {man }}} \frac{N_{e}}{120} \times \eta_{\text {vol }}\left(\frac{p_{\text {man }}}{\theta_{\text {man }}}, N_{e}\right)
$$

where $Q_{\text {eng }}$ is the incoming engine flow, $p_{\text {man }}$ and $\theta_{\text {man }}$ the manifold pressure and temperature, $V_{c y l}$ the engine displacement and $N_{e}$ the engine rotational speed. $\eta_{v o l}$ is a nonlinear function which is approximated using a second order polynomial calibrated on steady state test bench measurements.

At the outlet of the cylinders, the flow rate is the sum of $Q_{\text {eng }}$, the engine flow and $Q_{\text {fuel }}$, the fuel mass flow rate directly injected in the cylinders.

The exhaust pressure and temperature are respectively computed using (2) and from the inlet manifold gas temperature:

$$
\theta_{\text {avt }}=\theta_{\text {man }}+k_{\text {ech }} \frac{Q_{\text {fuel }} \times L H V}{C_{p}\left(Q_{\text {fuel }}+Q_{\text {eng }}\right)}
$$

where $L H V$ is the fuel lower heating value, $C_{p}$ the specific heat at constant pressure and $k_{e c h}$ represents the proportion of the total energy which is transferred to the flow at the exhaust:

$$
k_{e c h}=\Phi_{k_{e c h}}\left(N_{e}, Q_{f u e l}, Q_{e n g}\right)
$$

where $\Phi_{k_{e c h}}$ is a nonlinear function approximated by a second order polynomial calibrated on steady state test bench measurements.

\subsection{Actuator models}

The system has two inputs: the throttle and the wastegate, both considered as variable flow restrictions. The flow is computed using pressures on each side :

$$
\left\{\begin{array}{l}
Q_{m}=\frac{p_{u s}}{\sqrt{r \theta_{u s}}} S \sqrt{\gamma}\left(\frac{2}{\gamma+1}\right)^{\frac{\gamma+1}{2(\gamma-1)}} \quad \text { if } \quad \frac{p_{d s}}{p_{u s}} \leq\left(\frac{2}{\gamma+1}\right)^{\frac{\gamma}{\gamma-1}} \\
Q_{m}=\frac{p_{u s}}{\sqrt{r \theta_{u s}}} S\left(\frac{p_{d s}}{p_{u s}}\right)^{\frac{1}{\gamma}} \sqrt{\frac{2 \gamma}{\gamma-1}\left(1-\left(\frac{p_{d s}}{p_{u s}}\right)^{\frac{\gamma-1}{\gamma}}\right)} \text { otherwise }
\end{array}\right.
$$

where $S$ is the effective area of the orifice and depends nonlinearly on the actuators' position: $u_{p a p}$ and $u_{w g}$. The indices " $u s$ " and " $d s$ " respectively stand for upstream and downstream.

In order to reduce the number of states of the model, the dynamics of the actuators are neglected.

\subsection{Turbocharger model}

The turbocharger model relies on four static data-maps, extrapolated from manufacturers' data. The physics-based method that has been used is presented and validated in (El Hadef et al., 2012a, El Hadef et al., 2012b).

\subsubsection{Compressor and turbine sub-models}

The compressor and turbine mass flow rates are directly read from a nonlinear extrapolated data-map $\Phi_{Q_{m}}$ :

$$
Q_{m}=\Phi_{Q_{m}}\left(\pi, \omega_{t}\right)
$$

where $Q_{m}$ is the compressor (respectively turbine) mass flow rate and $\pi$ the compression (respectively expansion) ratio.

The flow temperature at the component outlet $\theta_{\text {out }}$ depends on the isentropic efficiency $\eta$ and is computed from the inlet flow temperature $\theta_{i n}$ :

$$
\theta_{\text {out }}=\theta_{\text {in }} \cdot h(\pi, \eta)
$$

where $h$ is a nonlinear function.

The isentropic efficiency is directly read from another extrapolated data-map $\Phi_{\eta}$ :

$$
\eta=\Phi_{\eta}\left(Q_{m}, \omega_{t}\right)
$$

\subsubsection{Fourth model state: turbocharger rotational speed}

The fourth state equation describes the turbocharger rotational speed $\omega_{t}$ and is given by:

$$
\dot{\omega}_{t}=\frac{1}{I}\left(T_{\text {turb }}-T_{\text {comp }}\right)
$$

where $I$ is the turbocharger inertia of the shaft which links the turbine to the compressor and $T_{\text {turb }}$ and $T_{\text {comp }}$ respectively represent the turbine and compressor torques.

Compressor and turbine torques depend on the mass flow rate, the inlet and outlet temperature and the turbocharger rotational speed. They are computed algebraically:

$$
\begin{aligned}
& T_{\text {comp }}=\frac{Q_{c o m p} \times C_{p} \times\left(\theta_{a p c}-\theta_{a m b}\right)}{\omega_{t}} \\
& T_{\text {turb }}=\frac{Q_{t u r b} \times C_{p} \times\left(\theta_{a v t}-\theta_{t u r b}\right)}{\omega_{t}}
\end{aligned}
$$

\subsection{Summary}

Globally, the model is nonlinear and has four states $p_{a p c}$, $p_{\text {man }}, p_{\text {avt }}$ and $\omega_{t}$. There are two control variables: $u_{p a p}$ and $u_{w g}$. In continuous time, it can be written as below: 


$$
\left\{\begin{array}{l}
p_{\text {ape }}^{\dot{ }}=\frac{\gamma r}{V_{\text {ape }}}\left(Q_{\text {comp }} \theta_{\text {apc }}-Q_{\text {thr }}\left(u_{\text {pap }}\right) \theta_{\text {ape }}\right) \\
p_{\text {man }}=\frac{\gamma r}{V_{\text {man }}} \theta_{\text {man }}\left(Q_{t h r}\left(u_{\text {pap }}\right)-Q_{\text {eng }}\right) \\
p_{\text {avt }}=\frac{\gamma r}{V_{\text {avt }}} \theta_{\text {avt }}\left(Q_{\text {eng }}+Q_{\text {fuel }}-Q_{t u r b}-Q_{w g}\left(u_{w g}\right)\right) \\
\dot{\omega_{t}}=\frac{1}{I}\left(T_{q_{\text {turb }}}-T_{q_{\text {comp }}}\right)
\end{array}\right.
$$

where $V_{\text {ape }}, V_{\text {man }}$ and $V_{a v t}$ respectively represent the volume between the compressor and the throttle, the volume of the intake manifold and the exhaust manifold volume (Fig. 1). $Q_{t h r}$ and $Q_{w g}$ stand for the throttle and wastegate flows, both obtained with (6).

For control design purposes, the model is discretized at a sampling time of $1 \mathrm{~ms}$, using Euler's backward differentiation method.

\section{NONLINEAR MODEL PREDICTIVE APPROACH}

\subsection{NMPC formulation}

MPC uses an iterative finite-time open loop optimization to compute the optimal actuator position vector $\mathrm{u}^{*}$ with respect to an objective function. At each time step, only the first command is applied to the real process. A new open-loop optimal problem is solved following the receding horizon principle (Camacho et al., 2004, Del Re et al., 2010, Ferreau et al., 2006).

Given the current system state $x_{0}$ and the vector of exogenous inputs $\sigma$ (principally the set points) at time instant $\mathrm{k}$, the discretized NMPC problem we address in this paper can be written as below:

$$
\begin{gathered}
\min _{u(.)} \sum_{i=k}^{k+N_{p}} J(x(i), y(i), u(i), \sigma) \\
x(k+1)=f(x(k), u(k), \sigma) \\
y(k)=g(x(k), u(k), \sigma) \\
\underline{x} \leq x(k) \leq \bar{x} \\
\underline{u} \leq u(k) \leq \bar{u} \\
x(k)=x_{0}
\end{gathered}
$$

where $x(i)$ denotes the system states and $u(i)$ stands for the vector of piecewise constant control inputs. $H_{p}=\left[k, k+N_{p}\right]$ is the so-called prediction horizon at time $k . f$ and $g$ are nonlinear functions describing the discrete-time system dynamics. Finally, $\underline{x}, \bar{x}, \underline{u}$ and $\bar{u}$ respectively stand for lower and upper bounds on the states and the control variables.

\subsection{Application to the turbocharged SI engine}

The air path control problem fits into the above formulation of an NMPC optimal control problem if we consider:

$$
\begin{gathered}
x:=\left(p_{\text {ape }}, p_{\text {man }}, p_{\text {avt }}, \omega_{t}\right)^{T} \\
y:=\left(p_{\text {man }}, p_{\text {avt }}\right) \\
u:=\left(u_{\text {pap }}, u_{w g}\right) \\
\sigma=\left(N_{e}, p_{\text {man }}^{S P}, p_{a m b}, \theta_{a m b}\right)
\end{gathered}
$$

The parameter $p_{\text {man }}^{S P}$ is used to track the desired manifold pressure set point. The right hand side of (15) is derived from the set of equations (13), while the output vector $y$ consists in the second and third states of the same model. No explicit state constraints are required. The manipulated variables are bounded in order to take into account the actuator saturations:

$$
0 \leq u \leq 100 \%
$$

As detailed before, the air path control of a turbocharged SI engine classically relies on using the same set point for the inlet manifold and compressor outlet pressures (Colin, 2006). It leads to the first objective function below:

$$
J_{1}=\alpha_{1}\left(p_{\text {man }}^{S P}-p_{\text {man }}\right)^{2}+\beta_{1}\left(p_{\text {man }}^{S P}-p_{\text {ape }}\right)^{2}
$$

where the weighting factors $\alpha_{1}$ and $\beta_{1}$ are used to scale and penalize each term of the cost function.

In this study, we propose to directly maximize the thermodynamic efficiency by minimizing the pressure difference between the inlet and outlet manifold. As such, a proper choice of the objective function is:

$$
J_{2}=\alpha_{2}\left(p_{\text {man }}^{S P}-p_{\text {man }}\right)^{2}+\beta_{2} \frac{p_{\text {avt }}}{p_{\text {man }}}
$$

where the weighting factors $\alpha_{2}$ and $\beta_{2}$ are used to scale and penalize each term of the cost function.

In both cases it is a multi-objective problem: if $\alpha_{i}$ is chosen too small compared to $\beta_{i}$, the inlet manifold pressure will not reach its desired value. In fact it would become more favorable to minimize the exhaust pressure than achieve the required inlet manifold pressure.

It can be seen that the NMPC formulation (14-19) avoids the use of a terminal penalty term and terminal constraints (Mayne et al., 2000). This design choice is principally due to the parameterization of the optimization problem in $\sigma$ which makes the definition of such additional terms intractable in this nonlinear framework. However, the closed-loop stability (in the sense of boundedness of state space trajectories) is ensured by the intrinsic dissipative properties of the system as well as the bounds on the control variables.

The only thing left is a proper choice of the control sampling time, which can be different from the model simulation sampling time. In order to control the fast dynamics of the engine as well as allow quick response to set point changes a new control is applied every $10 \mathrm{~ms}$.

\subsection{Prediction horizon and control variable partitions}

The control function $u(i)$ will be considered to be piecewise constant over the prediction horizon in order to get a finitedimensional optimization problem. On one hand the number of control variables should remain as small as possible in order to simplify the optimization problem. On the other hand, the degrees of freedom, represented by the number of partitions of $u(i)$, need to be adapted to the control horizon that is chosen. Otherwise, the MPC performances would be equivalent to the use of static data maps.

For the prediction horizon, the regular choice is made in relationship with the settling time of the system. In the case of a turbocharged engine, it has to be mentioned that the settling time is linked to the operating point of the engine. In particular, it depends on the engine and turbocharger rotational speed and can vary from about $80 \mathrm{~ms}$ to $250 \mathrm{~ms}$. In this study, a prediction horizon of $100 \mathrm{~ms}$ combined with a constant control value over this horizon has proved to be flexible enough to track realistic vehicle transients. The optimization problem is addressed in a classical single shooting framework using a simple trust-region reflective 
algorithm implemented in Matlab ${ }^{\circledR}$. This algorithm, initialized in the middle of the control variable space, converges within 10 iterations. The performances are depicted in the next section.

It can be seen that the simplicity of the problem, in particular the small number of model states and set points, as well as its fast convergence time match the requirements for a real-time implementation using the explicit MPC formulation. Such formulations will provide additional elements for the formal validation and verification of the closed-loop performance and stability. However, these explicit MPC constructions are beyond the scope of the present paper which concentrates in the remaining part on the analysis of the on-line optimizationbased MPC performances and discusses the sensitive aspects with respect to the design parameters.

\section{SIMULATION RESULTS}

\subsection{Model validation}

The model was calibrated using steady-state data acquired exclusively on an engine test bench. In this section we briefly present model validation results.
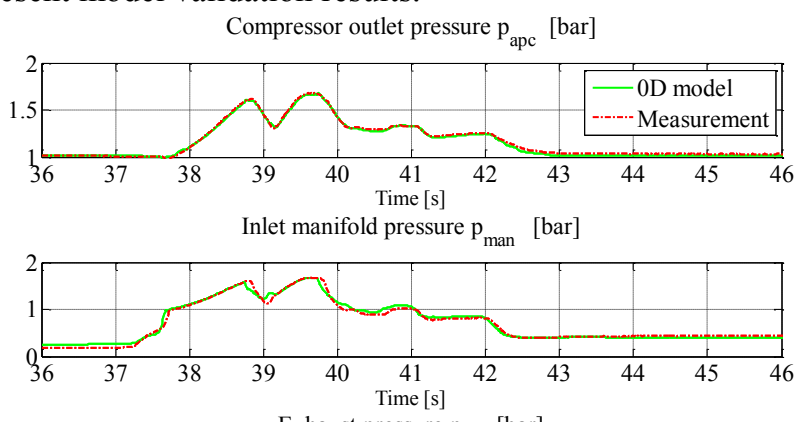
Exhaust pressure $\mathrm{p}_{\mathrm{avt}}[\mathrm{bar}]$
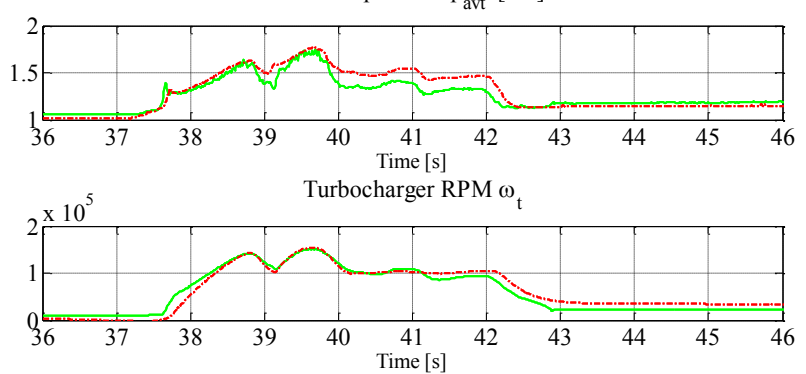

Fig. 4. Model states estimation validation example on vehicle transients. Over the complete validation cycle, engine speed varies from 1,000 to $6,000 \mathrm{rpm}$ while actuator positions vary from closed to fully open (including sudden openings).

The steady-state validation detailed in (El Hadef et al., 2012a) shows that the model prediction is very accurate over the entire engine operating range. The error remains well below $10 \%$ for most of the operating points. In Fig. 4 it can be seen that the model described above leads to accurate dynamic behaviour predictions which is compulsory for use in an MPC approach.

Altogether, steady-state and transient performances validate the modelling hypotheses as well as the different data that have been used. In particular, the exhaust pressure and the turbocharger rotational speed, computed thanks to the extrapolated compressor and turbine data-maps, are well predicted. It confirms that the recent physics-based extrapolation strategy presented in (El Hadef et al., 2012a, El Hadef et al., 2012b) leads to accurate results.

\subsection{Validation on vehicle transients}

In this section the performance of the NMPC is evaluated on an inlet manifold pressure transient. The complete transient represents 65 seconds of a driving sequence performed on a vehicle equipped with the engine depicted in this paper. In Fig. 5, we compare the performances obtained with the classical approach (i.e. tracking both compressor outlet and inlet manifold pressures reference trajectories) and with the new objective function (which directly maximizes the engine efficiency in the objective function).

It can be seen that both approaches lead to equivalent and satisfying tracking performances. Both pressure manifold signals remain in the $+/-100$ mbar tolerance interval required to achieve good drivability. On the actuator position graphs, note that, as expected, the classical approach leads, on average, to a wider throttle opening. However, the wastegate opening is usually greater with the new approach, designed to minimize the pumping losses. This can be clearly observed between 42.5 seconds and 44 seconds. This is also confirmed on the complete transient, for which averaged performances have been summed up in table 1 .

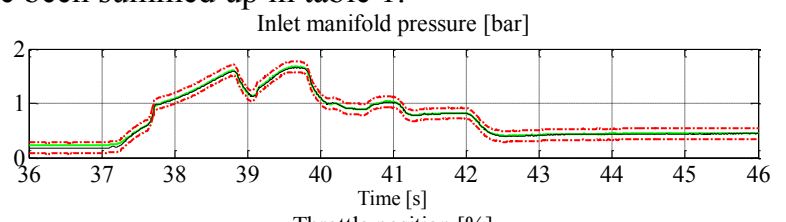

Throttle position [\%]

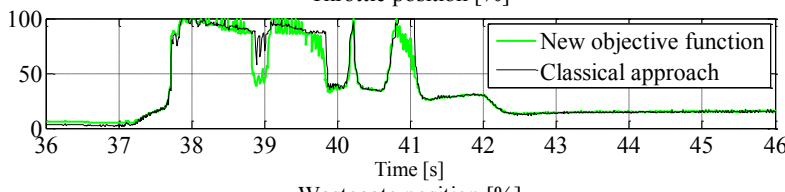

Wastegate position [\%]
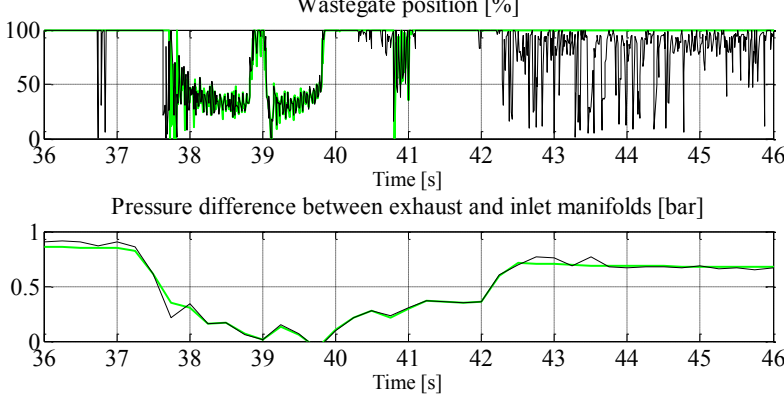

Fig. 5. Inlet manifold pressure tracking with corresponding actuator positions. The inlet manifold pressure reference trajectory is depicted through upper and lower $100 \mathrm{mbar}$ tolerance intervals (red dashed lines).

In Fig. 5, the pressure difference between exhaust and inlet manifold pressures is also compared for both approaches. In most cases the new objective function, designed to maximize the engine efficiency, leads to a smaller pressure difference, i.e. smaller pumping losses. In some rare cases, for example at time equal to 37.8 seconds, the classical approach leads to 
a smaller pressure drop. This is due to the calibration of the coefficients $\alpha_{\mathrm{i}}$ and $\beta_{\mathrm{i}}$ in (25) and (26). In fact, as explained before, they are used to penalize or not the pressure drop minimization with respect to the inlet manifold pressure tracking. At this particular point, the set of constant parameters that were chosen for the new objective function is probably more favorable to inlet manifold pressure tracking. However, on average, the new objective function leads to smaller pumping losses while maintaining equivalent inlet manifold pressure tracking performances. This is detailed in Table 1.

Table 1. Performances on a complete transient cycle

\begin{tabular}{|c|c|c|}
\hline Average values & $\begin{array}{c}\text { Classical } \\
\text { approach }\end{array}$ & $\begin{array}{c}\text { New objective } \\
\text { function }\end{array}$ \\
\hline Tracking error & $<20 \mathrm{mbar}$ & $<20 \mathrm{mbar}$ \\
\hline Pressure drop & $535 \mathrm{mbar}$ & $520 \mathrm{mbar}$ \\
\hline Throttle opening & $30 \%$ & $29 \%$ \\
\hline Wastegate opening & $75 \%$ & $80 \%$ \\
\hline Compressor efficiency & 0.70 & 0.71 \\
\hline Turbine efficiency & 0.56 & 0.57 \\
\hline
\end{tabular}

One can see that the throttle opening is maximized in the classical approach while it is the wastegate opening that is maximized with the new approach. The consequence of this is that, with the new objective function, the pressure difference between the exhaust and inlet manifold pressures is reduced by about $3 \%$ on the complete transient. The pumping losses are reduced in the same proportion. This can be explained by a slight increase in the compressor and turbine average efficiency.

\section{CONCLUSION}

In this paper, a NMPC approach is used to control the air path of a gasoline engine in a coordinated way. The main contribution resides in the combination of a MPC design with a nonlinear physical engine model. The main motivation to this multi-input approach is that it implicitly takes into account the nonlinear and coupled nature of the system. A second advantage is that it allows introducing physics-based terms in the objective function. In this study, a thermodynamic term replaces an empirical one in the objective function in order to maximize the engine efficiency. On this particular technical definition, the obtained pumping losses lead to a marginal effect on fuel consumption. However, the genuine advantage is that this formulation is independent of the tracking problem. In particular, the extension to gasoline engine torque tracking is straightforward. From an industrial point of view, the major advantage of such a generic design is the reduction of the calibration effort.

Finally, from the very beginning of this study, the real-time implementation constraints of the control have been taken into account. As such, the NMPC presented in this paper fulfills all the requisite criteria for online implementation using an explicit approach. The natural extension of this work is to compute its explicit form in order to validate the realtime performances (El Hadef et al., 2013).

\section{REFERENCES}

Bemporad, A., Morari, M., Dua, V., and Pistikopoulos, E. N. (2000). The Explicit Solution of Model Predictive Control via Multiparametric Quadratic Programming. In (ed.), American Control Conference, Chicago.

Camacho, E. F., and Bordons, C. (2004). Model Predictive Control. Springer, London.

Colin, G. (2006). Control of Fast Nonlinear Systems : Application to a Turbocharged SI Engine with Variable Valve Timing. PhD Thesis, University of Orleans.

Del Re, L., Glielmo, L., Guardiola, C., and Kolmanovsky, I. (2010). Automotive Model Predictive Control. Springer, Berlin.

Di Cairano, S., Bemporad, A., Kolmanovsky, I., and Hrovat, D. (2007). Model Predictive Control of Magnetically Actuated Mass Spring Dampers for Automotive Applications. Int. J. of Control, 80 (11), 1701-1716.

El Hadef, J., Colin, G., Chamaillard, Y., and Talon, V. (2012a). New Physics-Based Turbocharger Data-Maps Extrapolation Algorithms : Validation on a Spark-Ignited Engine. IFAC Workshop on Engine and Powertrain Control, Simulation and Modeling. France.

El Hadef, J., Colin, G., Chamaillard, Y., and Talon, V. (2012b). Physical-Based Algorithms for Interpolation and Extrapolation of Turbocharger Data Maps. SAE Int. J. Engines, 5 (2), 363-378.

El Hadef, J., Olaru, S., Rodriguez-Ayerbe, P., Colin, G., Chamaillard, Y., and Talon, V. (2013). Explicit Nonlinear Model Predictive Control of the Air Path of a Turbocharged Spark-Ignited Engine. 2013 IEEE Multi-Conference on Systems and Control. Hyderabad, India.

Eriksson, L. (2007). Modeling and Control of Turbocharged SI and DI Engines. Oil \& Gas Science and Technology - Rev. IFP Energies nouvelles, 62 (4), 523-538.

Eriksson, L., Nielsen, L., Brugard, J., and Bergström, J. (2002). Modeling of a Turbocharged SI Engine. Annual Reviews in Control, 26, 129-137.

Ferreau, H. J., Lorini, G., and Diehl, M. (2006). Fast Nonlinear Model Predictive Control of Gasoline Engine. In IEEE, P. O. T. (ed.), International Conference on Control Applications, Munich, Germany.

Grancharova, A., and Johansen, T. A. (2012). Explicit Nonlinear Model Predictive Control. Springer, Berlin.

Hendricks, E. (2001). Isothermal versus Adiabatic Mean Value SI Engine Models. 3rd IFAC Workshop, Advances in Automotive Control, 373-378.

Heywood, J. B. (1988). Internal Combustion Engines Fundamentals. McGraw-Hill.

Mayne, D. Q., Rawlings, J. B., Rao, C. V., and P.O.M., S. (2000). Constrained Model Predictive Control : Stability and Optimality. Automatica, 26 (6).

Moulin, P., Chauvin, J., and Youssef, B. (2008). Modelling and Control of the Air System of a Turbocharged Gasoline Engine. Proc. of the IFAC World Conference.

Pekar, J., Garimella, P., Germann, D., and Stewart, G. E. (2012). Experimental Results for Sensor Selection and Multivariable Controller Design for a Heavy-Duty Diesel Engine. IFAC Workshop on Engine and Powertrain Control, Simulation and Modeling. France. 